\title{
Bernstein Polynomials Method to Solve Fractional Model of Basic Enzyme Kinetics
}

\author{
Mohammed Alshbool \\ Department of Applied Mathematics, Abu Dhabi University, Abu Dhabi, United \\ Arab Emirates.
}

\begin{abstract}
Approximate solutions of fractional model of biochemical reaction using Bernstein polynomials method are derived in this paper. Enzyme-substrate reaction system is formed by a system of nonlinear ordinary differential equations. Fractional orders for the system are investigated at $\alpha=0.6, \alpha=0.75, \alpha=0.90$ and $\alpha=1$, to see the behavior of the system when the order is fraction between zero and one, $0<\alpha \leq 1$.
\end{abstract}

The effectiveness of the method is illustrated in a numerical experiment.

Keywords: Basic enzyme kinetics; Bernstein polynomials method. 


\section{Introduction}

$$
\begin{gathered}
E+A \stackrel{\mathrm{k}}{\longrightarrow} \longrightarrow \stackrel{\mathrm{k}_{2}}{\longrightarrow} E+X---{ }^{1} \\
\mathrm{k} \\
- \\
1
\end{gathered}
$$

scheme (1) is biochemical reaction model describe the enzyme processes and the basic enzymatic reaction where $E$ is the enzyme, The substrate, $Y$ the enzyme-substrate intermediate complex and $X$ the product [2]. The fractional time evolution of the scheme (1) can be determined from the solution of the system of fractional coupled nonlinear, given as

$$
\left\{\begin{array}{l}
x^{\alpha}(t)=-\epsilon x+\epsilon(x+\alpha-\lambda) y \\
y^{\alpha}(t)=x-(x+\alpha) y \\
z^{\alpha}(t)=\lambda y
\end{array}\right.
$$

with the boundary conditions

$$
x(0)=1, \quad y(0)=0, \quad z(0)=0
$$

Corresponding author. Email: alshbool.mohammed@gmail.com where $\epsilon, \alpha, \lambda$ are dimensionless parameters and $0<\alpha<1$.

Fractional order is investigated in the scheme, where $\alpha=0.6, \alpha=0.75, \alpha=0.90$.

Many numerical and analytical methods are applied to solve fractional differential equation such as homotopy perturbation method [3]. New modification in Bernstein functions is produced to solve fractional-order differential equations [1]. Basic enzyme kinetics is solved by many applied mathematical method such as variational iteration method [2] and multistage homotopy analysis method [7].

Our motivation for this work is to provide an accurate and efficient method to find the solution for the fractional biochemical Reaction Model. Bernstein polynomials method will be used to find the solutions, because Bernstein polynomials method is one of the important methods for solving differential equations $[4,5,6]$. Bernstein polynomials will be used to find the solutions of the model then correct the previous solutions by using the residual correction procedure. 


\section{Description of Method with Application}

We obtain an approximate solution of the problem (1) in the form of

$$
y_{n, \alpha}(x)=\sum_{k=0}^{n} c_{k} B_{n, k}^{\alpha}(x-c) .
$$

Here $0<\alpha<1, c_{k}, k=0,1,2, \ldots, n$ are the unknown Bernstein coefficients, $n$ is chosen for any positive integers, and $B_{k, n}{ }^{\alpha}(x)$ are obtained by putting $x \rightarrow x^{\alpha}$ in Bernstein polynomials as defined by

$$
B_{k, n}(x)=\left(\begin{array}{l}
n \\
k
\end{array}\right) \frac{x^{k}(R-x)^{n-k}}{R^{n}}, \quad k=0,1,2, \ldots, n \quad x \in[0, R], \quad i=0,1, \ldots, n
$$

it becomes

$$
B_{k, n}^{\alpha}(x)=\left(\begin{array}{l}
n \\
k
\end{array}\right) \frac{x^{k \alpha}\left(R-x^{\alpha}\right)^{n-k}}{R^{n}}, \quad k=0,1,2, \ldots, n \quad x \in[0, R], \quad i=0,1, \ldots, n .
$$

So

$$
y_{n, \alpha}(x)=\sum_{k=0}^{n} c_{k} B_{n, k}^{\alpha}(x-c)=C^{T} \phi^{*}(x) .
$$

By using some definitions and properties of the fractional calculus for the Caputo derivative, see [1], we have

$$
\begin{gathered}
D_{*}^{\alpha} c=0, \quad(c \text { constant }), \\
D_{*}^{\alpha} x^{\beta}=\left\{\begin{array}{l}
0, \quad \text { for } \beta \in N_{0} \\
\frac{\Gamma(\beta+1)}{\Gamma(\beta+1-\alpha)} x^{\beta-\alpha},
\end{array} \quad \text { and } \beta<\lceil\alpha\rceil,\right. \\
\text { for } \beta \in N_{0} \text { and } \beta \geq\lceil\alpha\rceil \text { or } \beta>\lfloor\alpha\rfloor .
\end{gathered}
$$

Then

$$
D^{\alpha} y(x) \approx C^{T} D^{\alpha} \phi *(x)
$$

where $D^{\alpha} y(x)$ is obtained in [1].

\section{Results and discussion}

The model (2) is solved for the case $m=5$ and $\lambda=0.5, \epsilon=0.6$. We study the fractional order of the model at and $\alpha=0.60, \alpha=0.75$ and $\alpha=0.90$, to see the behaviour of the model when the orders are not integer. Figure 1 and Tables $(1,2,3)$ show the results of our technique by using the Bernstein polynomials. 


\section{Conclusions}

In this paper we find the solutions of very important model. The fractional case of the model is investigated at deferent values of the fractional orders $\alpha$, the results are noted with more details values of $t$. These results show that the Bernstein polynomials method has much impact on the accuracy of the solution on this fractional basic enzyme kinetics scheme. The method is easily implemented.

\section{References}

[1] M.H.T. Alshbool, A.S. Bataineh, I. Hashim, and O. Isik. Solution of fractional-order differential equations based on the operational matrices of new fractional Bernstein functions. Journal of King Saud University-Science, 29:1-18, 2017.

[2] S. Goha, M. Noorani, and I. Hashim. Introducing variational iteration method to a biochemical reaction model. Nonlinear Analysis: Real World Applications, 11:2264$2272,2010$.

[3] S.H. Hosseinnia, A. Ranjibar, and S. Momani. Using an enhanced homotopy perturbation method in fractional differential equations via deforming the linear part. Appl. Math. Comput., 56:3138-3149, 2008.

[4] S. Khataybeh, I. Hashim, and M. Alshbool. Solving directly third-order odes using operational matrices of bernstein polynomials method with applications to fluid flow equations. Journal of King Saud University - Science, 2015.

[5] T. Kim, D. Kim, and J. Kwon. A note on degenerate bernstein polynomials. Journal of Inequalities and Applications, 129:10.1186/s13660-019-2071-1, 2019.

[6] A. Qasim and A. Hamed. Treating transcendental functions in partial differential equations using the variational iteration method with bernstein polynomials. Journal of Inequalities and Applications, 2019: https://doi.org/10.1155/2019/2872867, 2019.

[7] M. Zurigat, S. Momani, and A. Alawneh. The multistage homotopy analysis method: application to a biochemical reaction model of fractional order. International Journal of Computer Mathematics, 91:1030-1040, 2014. 

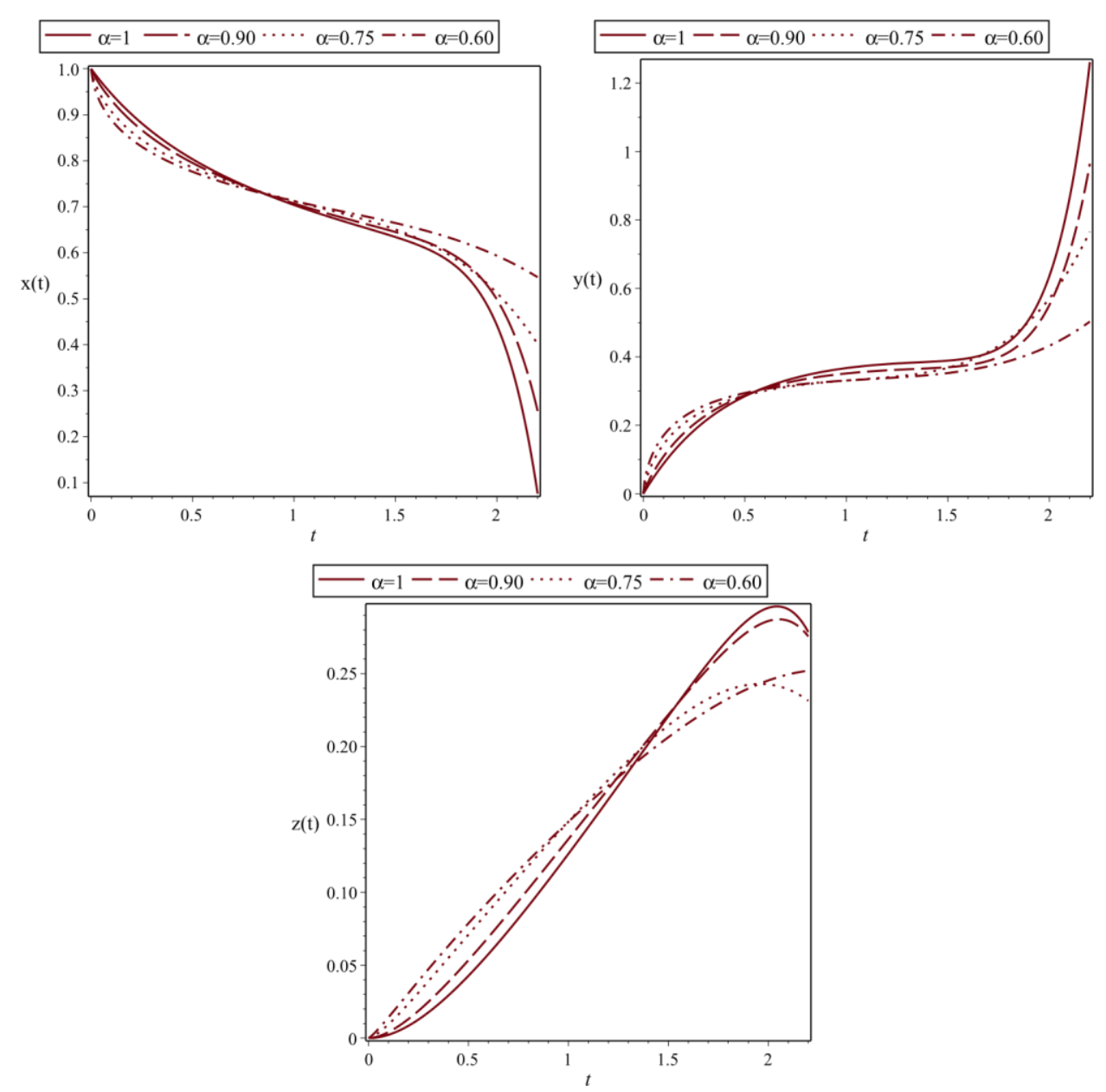

Figure 1: Compare between $\alpha=0.60, \alpha=0.75, \alpha=0.90$ and $\alpha=1$ to Examples 1, for the case $m=9$

Table 1: The values of the unknown $t$ for $x(t)$.

\begin{tabular}{ccccc}
\hline$t$ & $\alpha=0.60$ & $\alpha=0.75$ & $\alpha=0.90$ & $\alpha=1.0$ \\
\hline 0 & 1.00000000 & 1.00000000 & 1.00000000 & 1.00000000 \\
0.2 & 0.84621918 & 0.86254819 & 0.88569904 & 0.90091597 \\
0.4 & 0.79463294 & 0.80588512 & 0.82024957 & 0.83122466 \\
0.6 & 0.76029489 & 0.76717350 & 0.77367188 & 0.77909310 \\
0.8 & 0.73401764 & 0.73722000 & 0.73732984 & 0.73792557 \\
1.0 & 0.71242492 & 0.71242721 & 0.70718449 & 0.70384336 \\
1.5 & 0.66453641 & 0.65043810 & 0.64502633 & 0.63411080 \\
2.0 & 0.59338640 & 0.51218270 & 0.49783250 & 0.44162840
\end{tabular}


Table 2: The values of the unknown $t$ for $y(t)$.

\begin{tabular}{ccccc}
\hline$t$ & $\alpha=0.60$ & $\alpha=0.75$ & $\alpha=0.90$ & $\alpha=1.0$ \\
\hline 0 & $2.35 \times 10^{-69}$ & $2.90 \times 10^{-66}$ & 0.0000000 & 0.00000000 \\
0.2 & 0.22539585 & 0.20547978 & 0.17737174 & 0.15665013 \\
0.4 & 0.27892503 & 0.26848408 & 0.26101461 & 0.25198360 \\
0.6 & 0.30588001 & 0.30109891 & 0.30795855 & 0.31050745 \\
0.8 & 0.32141213 & 0.31978556 & 0.33540803 & 0.34613778 \\
1.0 & 0.33089118 & 0.33088291 & 0.35149498 & 0.36717898 \\
1.5 & 0.35270776 & 0.36808450 & 0.36972192 & 0.38894551 \\
2.0 & 0.43235950 & 0.57039590 & 0.55076340 & 0.63547201 \\
& & & &
\end{tabular}

Table 3: The values of the unknown $t$ for $z(t)$.

\begin{tabular}{ccccc}
\hline$t$ & $\alpha=0.60$ & $\alpha=0.75$ & $\alpha=0.90$ & $\alpha=1.0$ \\
\hline 0 & $8.422 \times 10^{-68}$ & $3.807 \times 10^{-66}$ & 0.00000000 & 0.00000000 \\
0.2 & 0.03090551 & 0.02360654 & 0.01312984 & 0.00848991 \\
0.4 & 0.06335338 & 0.05504070 & 0.03856943 & 0.02930861 \\
0.6 & 0.09362849 & 0.08694524 & 0.06925497 & 0.05767070 \\
0.8 & 0.12189179 & 0.11818109 & 0.10237549 & 0.09065292 \\
1.0 & 0.14840061 & 0.14840505 & 0.13653041 & 0.12641540 \\
1.5 & 0.20639822 & 0.21451866 & 0.22189207 & 0.22086967 \\
2.0 & 0.24532974 & 0.24263290 & 0.28611631 & 0.29514722
\end{tabular}

